\title{
Effect of Postnatal Chronic Lead Exposure on Spatial Learning and Memory in Male Rat
}

\author{
Maryam Bazrgar, Iran Goudarzi, ${ }^{1, *}$ Kataneh Abrari, ${ }^{1}$ Mahmoud Elahdadi Salmani, and \\ Taghi Lashkarbolouki ${ }^{1}$ \\ ${ }^{1}$ Faculty of Biology, Damghan University, Damghan, IR Iran \\ *Corresponding author: Iran Goudarzi, Faculty of Biology, Damghan University, Damghan, IR Iran. E-mail: irangoudarzi@du.ac.ir
}

Received: January 9, 2014; Accepted: February 28, 2014

\begin{abstract}
Background: Lead is a heavy metal with no apparent biological function. The widespread environmental contamination, the propensity to cause a wide spectrum of toxic effects and the number of individuals affected worldwide makes this ubiquitous neurotoxicant a public health problem of global magnitude. The nervous system is the primary target for the low levels of lead exposure and the developing brain appears to be especially vulnerable to lead neurotoxicity.

Objectives:The aim of present study was to evaluate the effects of neonatal lead exposure on spatial learning and memory in Morris water maze.

Materials and Methods: In this experimental study, 30 new born male rats were randomly divided into two groups: Control and lead exposure groups. Rat pups in lead exposure group received $8 \mathrm{mg} / \mathrm{kg}$ lead acetate intraperitoneally daily for 21 days. Then, rats trained with 6 trials per day for 6 consecutive days in the water maze at 36 th postnatal day (PD). Twenty four hours after ending of training, a probe test was done to measure memory retention.

Results: Results of this study demonstrated that exposure to lead could affect learning as indicating by significant differences in escape latency and traveled distance between 2 groups during 6 days of training. Lead exposure group exhibited significantly higher escape latency and distance traveled during training trials compared to control group. Also, lead exposure rats had higher platform location latency than control group during probe trial $(\mathrm{P}<0.05)$.

Conclusions: These data suggests that lead exposure during early postnatal period of brain development causes impairment in spatial learning and memory.
\end{abstract}

Keywords: Lead; Postnatal exposure; Learning; Memory; Rat

\section{Background}

Acute or chronic intoxication of animals and humans by lead is well documented. Lead is one of the most widely used metals in industries and in many countries exposure to lead continues to be a widespread problem. Animals and humans may get exposed to lead due to food or water contamination and to air pollution caused by industrial emission and combustion of lead-containing gasoline $[1,2]$. The toxicity of lead has been known for centuries, and symptoms caused by lead in the hematopoietic, gastrointestinal, urinary, cardiovascular, and nervous systems are well described [3, 4]. The central nervous system has been recognized as a primary target site for lead-induced toxicity [5]. It is also known that lead poisoning exerts its most severe consequences in the developing brain due to the immature blood-brain barrier and the absence of protein complexes able to sequester lead in mature tissue [6].

In humans, first two years of life is considered as the critical period as far as brain development is concerned.
In rats, first 4 weeks of life is considered as crucial period for brain development. During early period brain development, the brain undergoes major developmental and biochemical changes [7]. During this period, lead exposure can cause defective brain development and which results in neurological deficit $[7,8]$. These include diminished intelligence, reduced learning and memory capacities and deficient IQ scores in children $[9,10]$. Also animal study indicated similar neurological abnormalities and memory deficit following exposure to lead during gestation or developmental period [11]. Neurotoxic effects of lead on different site of brain were reported by several studies but the hippocampus has been the focus of much research on lead effects $[12,13]$. The hippocampus has been shown to be necessary for several types of learning and memory formation in rat and other mammals [14]. Also, it is reported that the hippocampus plays a particularly important role in processing and remembering of spatial and contextual information [15] and 
there is some evidence from animal studies that alternations in the hippocampus disrupt the animal's ability to learn spatial relations [16]. The developmental stages of different brain regions also determine the extent of lead impairment. In rat the neurogenesis of some areas of the brain is completed or nearly completed before day fifteen of gestation and others are well developed before birth (neocortex, limbic cortex), while the hippocampus and cerebellum is under development till early postnatal so that dentate gyrus cells of the hippocampus is produced largely postnatally in rodent $[17,18]$. Study has shown that N-methyl-D-aspartate (NMDA) and muscarinic cholinergic receptors are altered in the hippocampus of rats at 14 and 28 days of age, but not in older rats, that were exposed to lead continuously during development [12]. Also it is reported that protein kinase C (PKC) levels and activity were significantly altered in the hippocampus of lead-exposed developing rats [13].

\section{Objectives}

Taking this into consideration, the aim of present study was to evaluate spatial learning and memory by conducting Morris water maze test during infancy hood (on postnatal day 36 in rats that were exposed to lead acetate during early postnatal period of brain development.

\section{Materials and Methods}

The experimental protocol was approved by the Research and Ethics Committee of Damghan University. Adult female and male Wistar rats were obtained from the breeding colony of the Pasture Institute of Iran. They were housed in a temperature and light controlled room under a 12/12 hour light/dark cycle (light at 07:00 am) with food and water provided ad libitum. Female rats were housed overnight with males and checked on the following morning for the presence of copulation plugs. The day at which a vaginal plug found was used to define the beginning of gestation. Immediately after delivery, 30 male offspring were randomly divided into two different treatment groups including control and lead exposure groups. Rat pups in control group received distilled water intraperitoneally (i.p.) daily for 21 days and lead exposure group received $8 \mathrm{mg} / \mathrm{kg}$ lead acetate (Sigma) i.p. daily for 21 days. After wards animals of both groups were allowed to drink normal water till 36th postnatal day. The Male pups were weight from birth time until experiment time on a weekly basis.

\subsection{Morris Water Maze Task}

The Morris water maze used in our study was a black circular pool (140 cm in diameter, $60 \mathrm{~cm}$ high) filled with water (30 cm depth) at $24 \pm 2{ }^{\circ} \mathrm{C}$. The pool was divided into 4 quadrants of equal size. An invisible escape platform (10 cm diameter) was placed in the middle of one of the quadrants $(2 \mathrm{~cm}$ below the water surface) equidistant from the side wall and middle of the pool. The behavior of the animal (latency, distance and swim speed) was monitored by a video camera, mounted in the ceiling above the center of the pool, and a computerized tracking system (Ethovision; Noldus IT, The Netherlands). Four different starting positions were equally spaced around the perimeter of the pool. The training session consisted of six trials per day for 6 consecutive days which were started from one of the four start positions, used in a random sequence equal for every rat. A trial began by placing the rat into the water facing the wall of the pool at one of the starting points. If a rat failed to escape within 60 second, it was guided to the platform by the experimenter. Once the rat reached the platform, it was allowed to remain for 30 second and then placed in a holding cage for an inter-trial interval of 30 second. After the last trial, each animal was towel dried and returned to its home cage. Retention of the spatial training was assessed 24 hour after the last training session with a 60 second free-swim probe trial using a new starting position. The parameters measured on the probe trial were initial latency to cross the platform location, swimming speed and total swim distance.

\subsection{Statistical Analysis}

Data express as the mean \pm standard error of the mean (SEM). Statistical comparison was made through independent student t-test, using SPSS-16 and a statistical $\mathrm{P}$ value $\leq 0.05$ was considered significant.

\section{Results}

The cognitive function was assessed in the Morris water maze test. The mean escape latency and traveled distance for the trained rats decreased over the course of the 36 learning trials in 2 groups (Figure 1). Pair t-test analysis revealed significant differences between the 1st and 6th days of training in 2 groups for escape latency and traveled distance measure (Figure $1 \mathrm{~A}$ and $\mathrm{B}$ ). Lead exposure group exhibited significantly higher escape latency on 1st, 2nd, 3rd, 4th, 5th and 6th days ( $\mathrm{P}<0.05,0.01,0.001$, 0.001, 0.001 and 0.01, respectively) and distance traveled on 1st, 2nd, 3rd, 4th, 5th and 6th days $(\mathrm{P}<0.05,0.01,0.05$, 0.01, 0.01 and 0.05 , respectively) during training trials compared to control group. The analysis of swimming speed also showed no significant differences as training days progressed (Figure $1 \mathrm{C}$ ). The probe test was performed 24 hour after last acquisition trials and the data from probe trail is depicted in Figure $2 \mathrm{~A}-\mathrm{C}$. In probe trials, student t-test on platform location latency data indicated a significant difference among the 2 groups $(\mathrm{P}<0.05)$. To control for differences in the water maze performance, we recorded swimming speed of animals. Student t-test showed no significant differences of swimming speed between the 2 groups. Also, there were no significant differences on total distance traveled in the 2 groups (Figure $2 \mathrm{~B}$ ). 

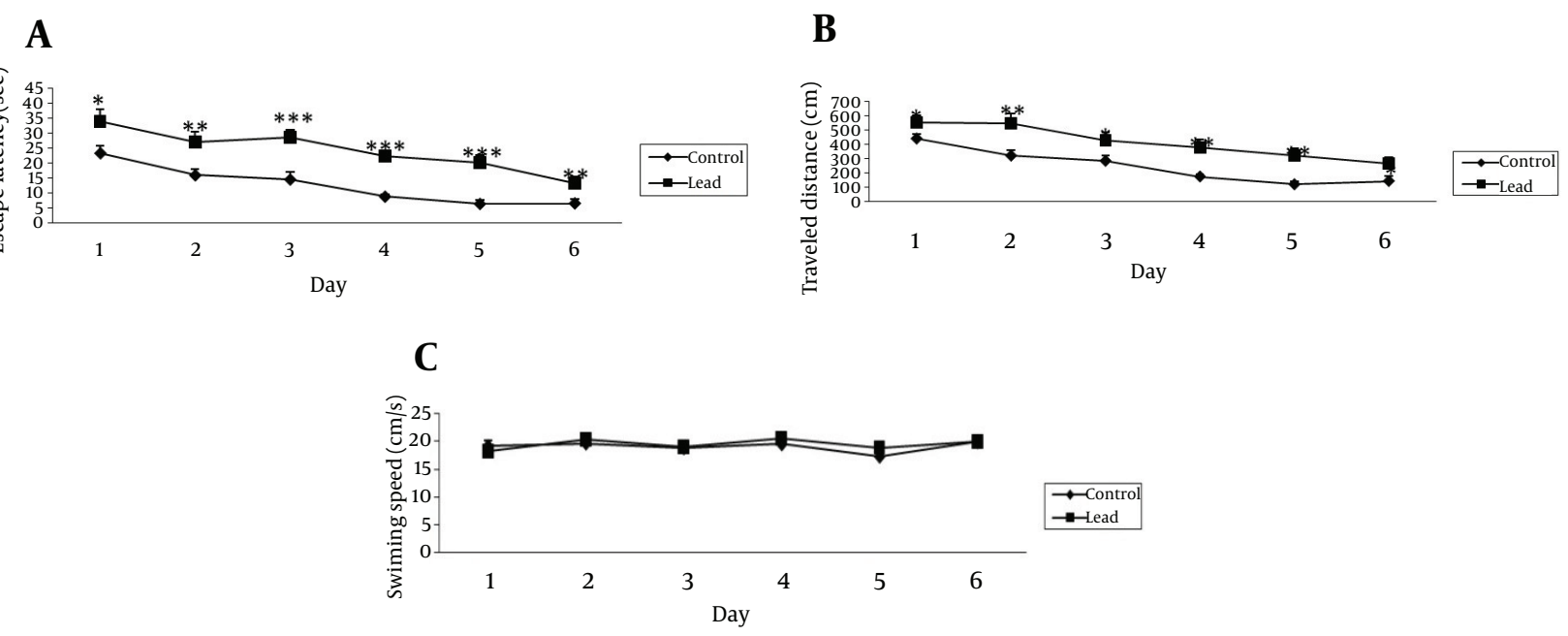

The figures show the escape latency and traveled distance significantly increased in lead exposed rats at the 1st, 2nd, 3rd, 4th, 5th and 6th days of training compared to the control group $\left({ }^{*} \mathrm{P}<0.05,{ }^{* *} \mathrm{P}<0.01,{ }^{* * *} \mathrm{P}<0.001\right.$, respectively). (C) There were no significant differences in swimming speed between lead exposed and control groups. Data express as mean \pm SEM of 15 animals in each groups.

Figure 2. Effects of Lead Treatment on Platform Location Latency (A), Swimming Speed (B) and Traveled Distance (C) in the Probe Trial on 7th Day

A

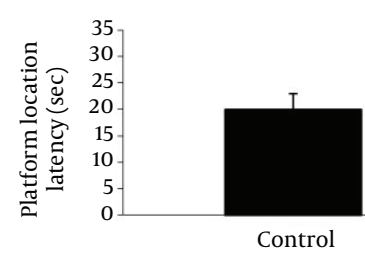

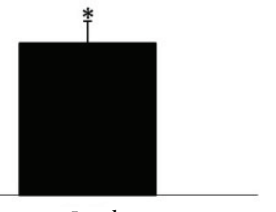

Lead
B

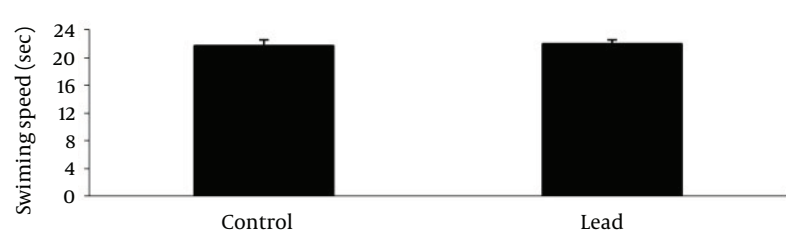

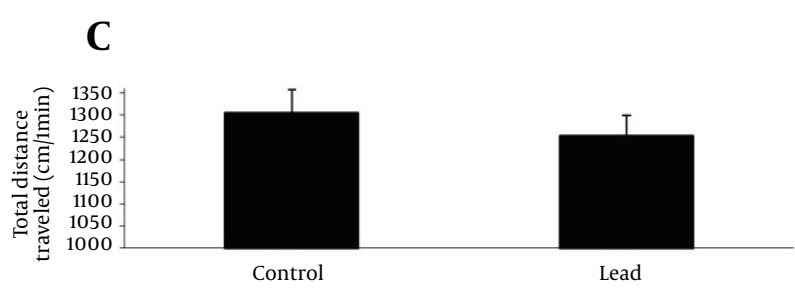

The figures show lead exposure group had higher platform location latency than control group. There were no significant differences in both total traveled distance and swimming speed in two groups. Data express as mean \pm SEM of 15 animals in each groups

\section{Discussion}

In the present study, we demonstrated that postnatal lead exposure resulted in significant learning and memory deficits in young adult offspring, as indicated by longer escape latency and traveled distance during training days compared to control group. Moreover, lead exposed animals showed significantly longer platform location latency than control animals in probe test. Swimming speed is considered a good measure of motor abilities in the context of the swimming task, and the general lack of any differences in swimming speed among the treatment groups indicates that motor skills were not impaired. Lead remains a serious toxic agent, particularly for children, despite reduction in the cases of exposure and the preventive measures taken worldwide. Several lines of evidence have revealed that lead exposure produces neurological damage and behavioral disruptions in human and in experimental animals. It is reported that exposure to lead during brain development results in behavioral alternation and learning and memory deficits [19, 20]. Previous studies reported that developmental lead exposure causes impair- 
ment in the acquisition phase in the Morris water maze $[14,21]$ radial arm maze task [22] and step-down inhibitory avoidance task [23]. This can be due to lead-induced impairments of the hippocampus [24]. Hippocampus plays an important role in both spatial and contextual learning. The Morris water maze tests the ability of rodents to learn and memorize the location of a hidden platform in a pool of water by cues keep around water mazes [25]. This task of spatial learning requires involvement of hippocampal NMDA and muscarinic cholinergic receptors [26]. Jett and Guilarte [12] found that developmental lead exposure causes alteration in NMDA and muscarinic cholinergic receptors in the hippocampus of rats at 14 days of age. Jaako-Movits et al. [27] established that exposure to lead during early development inhibits neurogenesis and alters the process of differentiation of new cells in the dentate gyrus of rat hippocampus, which could be responsible for the spatial memory impairments. Other studies have demonstrated that postnatal exposure to lead leads to disturbances in hippocampal structure, e.g., loss of granular and pyramidal cells as well as changes in dendritic spines [24, 28-30]. Also studies have demonstrated that lead affects cholinergic and serotonergic afferent connections to the hippocampus [31] and that change in hippocampal after discharge occur after neonatal lead exposure [32, 33]. In summary, we conclude that lead exposure during the critical period of early brain development affects spatial learning and memory. Future studies are needed to understanding mechanisms that will help us find a new way to prevent and treat the effects of lead toxicity on learning and memory.

\section{Acknowledgements}

We acknowledged of Damghan University for supporting work.

\section{Authors' Contributions}

All authors had equal role in design, work, statistical analysis and manuscript writing.

\section{Funding/Support}

\section{Damghan University.}

\section{References}

1. Pande M, Flora SJ. Lead induced oxidative damage and its response to combined administration of alpha-lipoic acid and succimers in rats. Toxicology. 2002;177(2-3):187-96.

2. Mudipalli A. Lead hepatotoxicity \& potential health effects. Indian J Med Res. 2007;126(6):518-27.

3. Goyer RA. Lead toxicity: current concerns. Environ Health Perspect. 1993;100:177-87.

4. Aggarwal HK, Yashodara BM, Nand N, Sonia. Chakrabarti D, Bharti K. Spectrum of renal disorders in a tertiary care hospital in Haryana. J Assoc Physicians India. 2007;55:198-202.

5. Silbergeld EK. Mechanisms of lead neurotoxicity, or looking beyond the lamppost. FASEB J.1992;6(13):3201-6.

6. Goyer RA. Lead toxicity: from overt to subclinical to subtle health effects. Environ Health Perspect. 1990;86:177-81.
7. Rice D, Barone SJ. Critical periods of vulnerability for the developing nervous system: evidence from humans and animal models. Environ Health Perspect. 2000;108 Suppl 3:511-33.

8. Brent RL, Tanski S, Weitzman M. A pediatric perspective on the unique vulnerability and resilience of the embryo and the child to environmental toxicants: the importance of rigorous research concerning age and agent. Pediatrics. 2004;113(4 Suppl):935-44.

9. Bellinger D, Leviton A, Waternaux C, Needleman H, Rabinowitz $M$. Longitudinal analyses of prenatal and postnatal lead exposure and early cognitive development. $N$ Engl J Med. 1987;316(17):1037-43.

10. Dietrich KN, Berger OG, Succop PA, Hammond PB, Bornschein RL The developmental consequences of low to moderate prenatal and postnatal lead exposure: intellectual attainment in the Cincinnati Lead Study Cohort following school entry. Neurotoxicol Teratol.1993;15(1):37-44.

11. Cohn J, Cox C, Cory-Slechta DA. The effects of lead exposure on learning in a multiple repeated acquisition and performance schedule. Neurotoxicology. 1993;14(2-3):329-46.

12. Jett DA, Guilarte TR. Developmental lead exposure alters Nmethyl-D-aspartate and muscarinic cholinergic receptors in the rat hippocampus: an autoradiographic study. Neurotoxicology. 1995;16(1):7-18.

13. Farmer SJ. Inhibition of protein kinase C (PKC) activity in hippocampal fractions of leadexposedrats. Toxicol.1995;15(1):259.

14. Soodi M, Naghdia N, Sharifzadeh M, Ostad SN, Abdollahi M. Effect of lead $(\mathrm{Pb} 2+)$ exposure in female pregnant rats and their offspring on spatial learning and memory in Morris water maze. Iran J Pharm Res. 2008;7(1):43-51.

15. Jarrard LE. On the role of the hippocampus in learning and memory in the rat. Behav Neural Biol.1993;60(1):9-26.

16. Olton DS, Markowska AL. Memory and hippocampal function as targets for neurotoxic substances. Neurotoxicology. 1994;15(3):439-43.

17. Xu SZ, Bullock L, Shan CJ, Cornelius K, Rajanna B. PKC isoforms were reduced by lead in the developing rat brain. Int J Dev Neurosci. 2005;23(1):53-64.

18. Rodier PM. Chronology of neuron development: animal studies and their clinical implications. Dev Med Child Neurol. 1980;22(4):525-45.

19. Yang Y, Ma Y, Ni L, Zhao S, Li L, Zhang J, et al. Lead exposure through gestation-only caused long-term learning/memory deficits in young adult offspring. Exp Neurol. 2003;184(1):489-95.

20. Moreira EG, Rosa GJ, Barros SB, Vassilieff VS, Vassillieff I. Antioxidant defense in rat brain regions after developmental lead exposure. Toxicology. 2001;169(2):145-51.

21. Jett DA, Kuhlmann AC, Farmer SJ, Guilarte TR. Age-dependent effects of developmental lead exposure on performance in the Morris water maze. Pharmacol Biochem Behav.1997;57(1-2):271-9.

22. Munoz C, Garbe K, Lilienthal H, Winneke G. Neuronal depletion of the amygdala resembles the learning deficits induced by low level lead exposure in rats. Neurotoxicol Teratol.1989;11(3):257-64.

23. Chen H, Ma T, Ho IK. Effects of developmental lead exposure on inhibitory avoidance learning and glutamate receptors in rats. Environ Toxicol Pharmacol. 2001;9(4):185-91.

24. Petit TL, Alfano DP, LeBoutillier JC. Early lead exposure and the hippocampus: a review and recent advances. Neurotoxicology. 1983;4(1):79-94.

25. Morris RG, Garrud P, Rawlins JN, O'Keefe J. Place navigation impaired in rats with hippocampal lesions. Nature. 1982;297(5868):681-3.

26. McNamara RK, Skelton RW. The neuropharmacological and neurochemical basis of place learning in the Morris water maze. Brain Res Brain Res Rev.1993;18(1):33-49.

27. Jaako-Movits K, Zharkovsky T, Romantchik O, Jurgenson M, Merisalu E, Heidmets LT, et al. Developmental lead exposure impairs contextual fear conditioning and reduces adult hippocampal neurogenesis in the rat brain. Int $J$ Dev Neurosci. 2005;23(7):627-35.

28. Campbell JB, Woolley DE, Vijayan VK, Overmann SR. Morphometric effects of postnatal lead exposure on hippocampal development of the 15-day-old rat. Brain Res.1982;255(4):595-612. 


\section{BazrgarM et al.}

29. Slomianka L, Rungby J, West MJ, Danscher G, Andersen AH. Dosedependent bimodal effect of low-level lead exposure on the developing hippocampal region of the rat: a volumetric study. Neurotoxicology.1989;10(2):177-90.

30. Kiraly E, Jones DG. Dendritic spine changes in rat hippocampal pyramidal cells after postnatal lead treatment: a Golgi study. Exp Neurol.1982;77(1):236-9.

31. Alfano DP, Petit TL. Behavioral effects of postnatal lead exposure: possible relationship to hippocampal dysfunction. Behav Neural Biol. 1981;32(3):319-33.

32. McCarren M, Eccles CU. Neonatal lead exposure in rats: II. Effects on the hippocampal afterdischarge. Neurobehav Toxicol Teratol. 1983;5(5):533-40.

33. McCarren M, Young GA, Eccles CU. Spectral analysis of kindled hippocampal afterdischarges in lead-treated rats. Epilepsia. 1984;25(1):53-60. 